\title{
Up-to-date information on polymyxin B-immobilized fiber column direct hemoperfusion for septic shock
}

\author{
Chieko Mitaka ${ }^{1}$, Makio Kusaoi $^{2}$, Izumi Kawagoe ${ }^{1}$, Daizoh Satoh ${ }^{1}$ \\ Departments of ${ }^{1}$ Anesthesiology and Pain Medicine and ${ }^{2}$ Internal Medicine and Rheumatology, Juntendo University Faculty of Medicine, Tokyo, Japan
}

Endotoxin adsorption therapy by polymyxin B-immobilized fiber column direct hemoperfusion (PMX-DHP) has been used for the treatment of septic shock patients. Endotoxin, an outer membrane component of Gram-negative bacteria, plays an important role in the pathogenesis of septic shock. Endotoxin triggers a signaling cascade for leukocytes, macrophage, and endothelial cells to secrete various mediators including cytokines and nitric oxide, leading to septic shock and multiple organ dysfunction syndrome. PMX-DHP directly adsorbed not only endotoxin but also monocytes and anandamide. It reduced blood levels of inflammatory cytokines such as interleukin (IL)-1, IL-6, tumor necrosis factor-alpha and IL-17A, adhesion molecules, plasminogen activator inhibitor 1, and high mobility group box-1. As a result, PMX-DHP increased blood pressure and reduced the dose of vasoactive-inotropic agents. PMX-DHP improved monocyte human leukocyte antigen-DR expression in patients with severe sepsis and septic shock. A post hoc analysis of EUPHRATES (Evaluating the Use of Polymyxin B Hemoperfusion in Randomized Controlled Trial of Adults Treated for Endotoxemia and Septic Shock) trial has shown that PMX-DHP significantly reduced 28-day mortality compared with the control group in septic shock patients with endotoxin activity assay level between 0.60 and 0.89 . Longer duration of PMX-DHP may be another strategy to bring out the beneficial effects of PMX-DHP. Further studies are needed to confirm the efficacy of PMX-DHP treatment for septic shock.

Key Words: duration of therapy; endotoxin; hemoperfusion; polymyxin B; sepsis; septic shock

\section{INTRODUCTION}

Endotoxin adsorption therapy by polymyxin B-immobilized fiber column direct hemoperfusion (PMX-DHP) has been used for the treatment of septic shock patients in Japan since 1994. Indications of PMX-DHP are (1) endotoxemia or Gram negative-bacterial infection is suspected; (2) systemic inflammatory response syndrome (SIRS); and (3) septic shock patients who needs vasopressor-inotropic agents. Its use continues to widen worldwide year by year. This review evaluated the mechanism of PMX-DHP treatment and effects of PMX-DHP treatment on hemodynamics, mediators and pulmonary oxygenation and offered information on prospective multicenter randomized controlled trial (RCT) of PMX-DHP, longer duration of PMX-DHP in patients with septic shock, and cost-effectiveness of PMX-DHP for septic shock.

\section{Review Article}

Received: February 2, 2021

Revised: March 2, 2021

Accepted: March 3, 2021

Corresponding author

Chieko Mitaka

Department of Anesthesiology and Pain Medicine, Juntendo University Faculty of Medicine, 3-1-3 Hongo, Bunkyo-ku, Tokyo 113-8431, Japan Tel: +81-3-3813-3111

Fax: +81-3811-7285

E-mail: c-mitaka@juntendo.ac.jp

Copyright () 2021 The Korean Society of Critical Care Medicine

This is an Open Access article distributed under the terms of Creative Attributions Non-Commercial License (https:/I creativecommons.org/li-censes/by-nc/4.0/) which permits unrestricted noncommercial use, distribution, and reproduction in any medium, provided the original work is properly cited. 


\section{MECHANISM OF PMX-DHP TREATMENT FOR SEPTIC SHOCK}

\section{Direct Adsorption of Circulating Endotoxin}

The major mechanism of PMX-DHP is the removal of circulating endotoxin (Figure 1). The covalent binding of polymyxin B onto the surface of the polystyrene-based carrier fiber in PMX-DHP inactivates the endotoxin in the blood without exerting toxicity. Endotoxin (lipopolysaccharide [LPS]), an outer membrane component of Gram-negative bacteria, plays an important role in the pathogenesis of septic shock. LPS is transported in a complex with a carrier protein, LPS-binding protein (LBP). The LPS-LBP complex exerts biological activity via CD14 on the monocyte and macrophage surface. Full activation of the Toll-like receptor 4 (TLR4) requires the membrane receptor CD14 and an accessory protein, myeloid differentiation 2 (MD2). LPS-LBP complex is transferred to TLR4 and TLR4 complex recruits myeloid differentiation factor 88 (MyD88) and interleukin (IL)-1 receptor-associated kinase (IRAK) [1]. Then, signaling pathways activate nuclear factor kappa B (NF-kB), which induces the production of proinflammatory cytokines such as IL-1, IL-6, and tumor necrosis factor

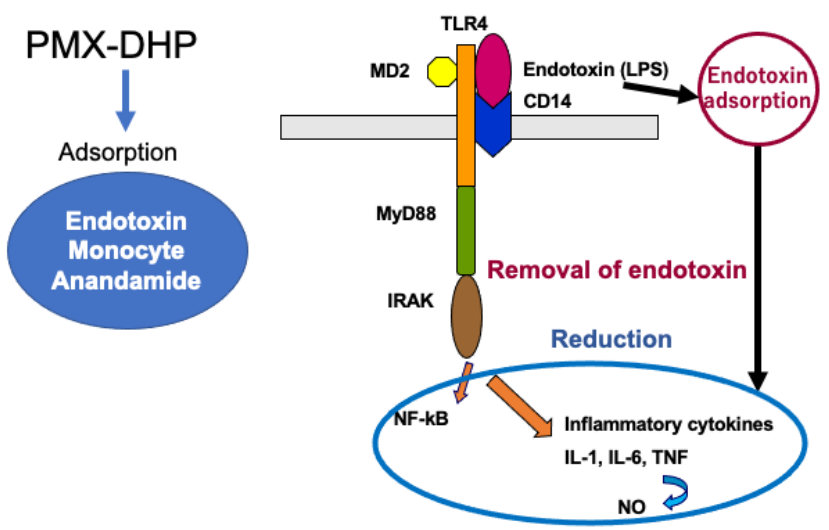

Figure 1. Recognition of endotoxin (lipopolysaccharide [LPS]) on the surface of phagocytes and mechanism of polymyxin B-immobilized fiber column direct hemoperfusion (PMX-DHP) treatment. LPS is opsonized by LPS-binding protein (LBP), and LPS-LBP complex is recognized by CD14, on the monocyte and macrophage surface. LPS-LBP-CD14 ternary complex activates TLR4, which in turn signals through My88 and interleukin (IL)-1 receptor-associated kinase (IRAK). Signaling pathways activate nuclear factor kappa B (NF-kB), which induces the production of proinflammatory cytokines and nitric oxide (NO). PMX-DHP directly adsorbs endotoxin and reduces circulating level of endotoxin. In addition, PMX-DHP adsorbs monocyte and anandamide. As a result, inflammatory cytokines and NO decrease. TLR4: Toll-like receptor 4; MD2: myeloid differentiation 2; MyD88: myeloid differentiation factor 88; TNF: tumor necrosis factor.

\section{KEY MESSAGES}

- Polymyxin B-immobilized fiber column direct hemoperfusion (PMX-DHP) directly adsorbed not only endotoxin but also monocytes and anandamide.

- PMX-DHP increased blood pressure and reduced the dose of vasoactive-inotropic agents.

- Longer duration of PMX-DHP may be another strategy to bring out the beneficial effects of PMX-DHP.

(TNF)-alpha and nitric oxide (Figure 1). Endothelial activation induces adhesion molecules such as endothelial leukocyte adhesion molecule-1, intercellular adhesion molecule-1 and vascular cell adhesion molecule-1, which result in leukocyte rolling and leukocyte adhesion [2].

\section{Additional Adsorption Other Than Endotoxin}

PMX-DHP has been shown to reduce the number of CD16 ${ }^{+}$$\mathrm{CD} 14^{+}$monocytes and decrease the monocyte expression of TLR4 in patients with septic shock [3]. CD16 ${ }^{+} \mathrm{CD} 14^{+}$monocytes mean activated cells and cause the immunosuppressin in severe infection. The adsorption of $\mathrm{CD} 6^{+} \mathrm{CD} 14^{+}$monocytes by PMX-DHP treatment improves the clinical outcome in septic shock patients. The adsorption of $\mathrm{CD} 16^{+} \mathrm{CD} 14^{+}$monocytes may be the mechanism by which PMX-DHP treatment improves the clinical outcome in patients with septic shock. In fact, Nishibori et al. [4] have revealed that PMX-DHP column specifically binds monocytes from the peripheral blood leukocytes of septic patients by using immunocytochemical and electron microscopic techniques. The removal of monocytes from septic patients may have beneficial effects by reducing the interaction between monocytes and functionally associated cells, such as vascular endothelial cells. Although the specific mechanism still remains unknown, PMX-DHP may remove monocytes by adsorbing the LPS-monocytes complex. In addition, anandamide, an endogenous cannabinoid, is generated by macrophages during endotoxin shock, and is thought to contribute to hypotension in a paracrine fashion. Wang et al. [5] have reported that PMX-DHP directly bound to anandamide and neutralized bioactivity effect such as vasodilation conferred by anandamide.

\section{Effect of PMX-DHP on Hemodynamics}

Lots of studies have reported that PMX-DHP helps to increase blood pressure in patients with sepsis [6-15]. The dose of vasoactive-inotropic agents was consistently decreased after PMXDHP treatment $[6,10,11,13,14,16]$. Our study suggested that 
PMX-DHP treatment is particularly useful for improving hemodynamics in septic shock patients with excessive dilated peripheral blood vessels [16]. The key mechanism of this finding may be due to reduction in vasodilatory mediators such as anandamide and nitric oxide by PMX-DHP. Because, PMXDHP directly adsorbed anandamide [5] and reduced nitric oxide in septic patients [17].

\section{Effect of PMX-DHP on Mediators}

PMX-DHP decreased blood levels of IL-6 [8,11,12,15,18,19], IL-8 [18], IL-10 [12], TNF-alpha [8,12], plasminogen activator inhibitor $1[12,13,18]$, neutrophil elastase $[18,20]$, soluble endothelial leukocyte adhesion molecule-1 [14], and high mobility group box-1 (HMGB-1) [15]. In a subset of patients with liver failure [19], HMGB-1 level reduced after PMX-DHP treatment in survivors, but HMGB-1 level increased after PMX-treatment in non-survivors. PMX-DHP treatment significantly $(\mathrm{P}<$ $0.01)$ reduced urinary nitric oxide breakdown products nitrate and nitrite (NOx) in septic patients compared with conventional treatment [17].

Coudroy et al. [21] assessed the influence of PMX-DHP on plasma cytokine levels in ancillary study of ABDOMIX trial. The plasma levels of TNF-alpha, IL-1 alpha, IL-1 beta, IL-1 receptor antagonist (RA), IL-4, IL-6, IL-10, IL-17A, IL-22, and interferon-gamma were measured in 213 patients with peritonitis-induced septic shock enrolled in the randomized trial ABDOMIX trial. Plasma levels of TNF-alpha, IL-1 beta, IL-6, IL-10, and IL-1RA decreased significantly $(\mathrm{P}<0.0001)$ in both groups. IL-17A decreased significantly $(\mathrm{P}=0.04)$ in the PMXDHP group compared with the control group. IL-17 is a proinflammatory cytokine produced by immune cells during sepsis and plays roles in innate immune responses to bacteria and fungi [22]. Since inhibition of IL-17A was protective in mice sepsis model induced by cecal ligation and puncture [23], PMXDHP might have protective effect by decreasing IL-17A level in septic shock. However, several proinflammatory and antiinflammatory cytokines except IL-17A did not differ between the two groups.

PMX-DHP significantly decreased the proapoptotic activity of the plasma of septic patients on cultured renal cells [24]. In addition, Sequential Organ Failure Assessment (SOFA) score and risk, injury, failure, loss and end-stage (RIFLE) criteria were significantly $(\mathrm{P}=0.02, \mathrm{P}=0.04$, respectively $)$ improved 72 hours after PMX-DHP treatment [25]. These findings suggest that protective effects of PMX-DHP against acute kidney injury may be attributable to a reduced systemic proapoptotic activity.

\section{Effect of PMX-DHP on Monocyte Human Leukocyte Antigen-DR}

The surface antigen, major histocompatibility complex class II, human leukocyte antigen (HLA)-DR allows antigen presentation to T cells and is essential for the initiation of the immune cascade during sepsis. Ono et al. [26] have shown that monocyte HLA-DR extremely decreased in patients with septic shock, and that the monocyte HLA-DR expression significantly increased after PMX-DHP treatment. Monneret et al. [27] have demonstrated that survivors had increased monocyte HLA-DR, while non-survivors had not in patients with septic shock, suggesting that monocyte HLA-DR is an independent predictor of mortality in patients with septic shock.

Recently, Srisawat et al. [28] conducted prospective RCT between in severe sepsis patients with endotoxin activity assay (EAA) level $\geq 0.6$ to elucidate efficacy of PMX-DHP treatment on change in monocyte HLA-DR expression. The median change in monocyte HLA-DR from baseline to day 3 was significantly $(\mathrm{P}=0.027)$ higher in the PMX-DHP group than the non-PMX-DHP group. These findings suggest that PMXDHP treatment may improve immunoparalysis status by improving monocyte HLA-DR expression in patients with severe sepsis and septic shock.

\section{Effect of PMX-DHP on Pulmonary Oxygenation}

The studies showed that the $\mathrm{PaO}_{2} / \mathrm{FIO}_{2}$ ratio increased after PMX-DHP treatment in most of the studies [8,10,12-14]. S100$\mathrm{A} 12$, also known as $\mathrm{S} 100$ calcium-binding protein $\mathrm{A} 12$ is a protein that in humans is encoded by the S100A12 gene. S100A12 is useful as early marker of acute lung injury. Wittkowski et al. [29] have shown that neutrophil-derived S100A12 was found at high concentration in pulmonary tissue and bronchoalveolar lavage fluid in acute lung injury. The authors concluded that S100A12 expression may reflect neutrophil activation during lung inflammation and contribute to pulmonary inflammation and endothelial activation. Takahashi et al. [30] have demonstrated that S100A12 significantly decreased after PMXDHP treatment and $\mathrm{PaO}_{2} / \mathrm{FiO}_{2}$ ratio significantly improved.

\section{PROSPECTIVE MULTICENTER RCT}

\section{Pilot-Controlled Study of PMX-DHP}

Vincent et al. [9] performed the first prospective RCT of the PMX-DHP treatment in 36 patients with severe sepsis or septic shock in six academic centers in Europe. In the PMX-DHP group, mean arterial pressure increased significantly $(\mathrm{P}=0.006)$ from baseline to day 2 without major side effect. Cardiac in- 
dex, left ventricular stroke work index, and oxygen delivery were significantly $(\mathrm{P}<0.05)$ greater in the PMX-DHP group than in the control group. In addition, the need for continuous renal replacement therapy was decreased in the PMX-DHP group. However, there were no significant differences in the change in endotoxin and IL-6 levels from baseline to 6-8 hours after treatment or to 24 hours after treatment between the two groups. In this study, PMX-DHP was applied to patients only once. One session of PMX-DHP might be insufficient in cases with overwhelming endotoxin into circulation.

\section{EUPHAS Trial}

Early Use of Polymyxin B Hemoperfusion in Abdominal Septic Shock (EUPHAS) trial conducted at 10 Italian intensive care units (ICUs) between December 2004 and December 2007 [10]. This trial enrolled 64 patients with severe sepsis or septic shock who underwent emergency surgery for intra-abdominal infection. The primary end points were changes from baseline to 72 hours in mean arterial pressure and vasopressor requirement. The secondary end points were $\mathrm{PaO}_{2} / \mathrm{FiO}_{2}$ ratio, changes in SOFA score, and 28-day mortality. Thirty-four patients in the PMX-DHP group and 30 patients in the conventional therapy group were enrolled in the study. This trial was early terminated by the ethics committee because of a significant mortality reduction in the PMX-DHP group. Mean arterial pressure significantly $(\mathrm{P}=0.001)$ increased and inotropic score significantly $(\mathrm{P}<0.001)$ decreased after 72 hours in the PMX-DHP group. The vasopressor dependency index decreased significantly $(\mathrm{P}<0.001)$ in the $\mathrm{PMX}$-DHP group. $\mathrm{PaO}_{2} / \mathrm{FiO}_{2}$ ratio significantly $(\mathrm{P}=0.049)$ increased in the PMX-DHP group. At 72 hours, the PMX-DHP group had a greater reduction in SOFA score compared with the conventional therapy group. The 28-day mortality was $32 \%$ in the PMX-DHP group and $53 \%$ in the conventional therapy group. However, the conclusions of this study should be taken with caution, since the trial was prematurely stopped.

\section{ABDOMIX Trial}

ABDOMIX trial conducted at 18 ICUs in France from October 2010 to March 2013. This trial enrolled 243 patients with septic shock within 12 hours after emergency surgery for peritonitis related to organ perforation [31]. Thirty-three of 119 patients (27.7\%) died at day 28 in the PMX-DHP group and 22 of 113 patients (19.5\%) in the control group. There were no significant differences in 28-day mortality and 90-day mortality between the two groups. In addition, there were no differences in hemodynamic patterns and organ failure evolution between the two groups. Decrease in platelet count at day 3 was more frequent in the PMX-DHP group than in the control group $(\mathrm{P}=0.002)$. There were only 81 of 119 patients $(69.8 \%)$ who received two sessions of PMX-DHP treatment due to circuit clotting, technical problems, death or major hemodynamic instability.

\section{EUPHRATES Trial}

The EUPHRATES (Evaluating the Use of Polymyxin B Hemoperfusion in a RCT of Adults Treated for Endotoxemia and Septic Shock) trial conducted at 55 ICUs in the United States and Canada between September 2010 and June 2017 [32]. This trial enrolled 450 adult septic shock patients with an EAA level $\geq 0.6$ and multiple organ dysfunction score (MODS) $>9$. The patients were divided into two groups: PMX-DHP group $(\mathrm{n}=224)$ and sham group $(\mathrm{n}=226)$. This trial was blinded with sham perfusion treatments. Among 450 eligible enrolled patients, 449 completed the study. In the PMX-DHP group, two sessions of PMX-DHP treatments (1.5-2 hours) were performed. PMXDHP treatment compared with sham hemoperfusion did not significantly reduce 28 -day mortality, $37.7 \%$ vs. $34.5 \%$, respectively. In the MODS $>9$ patients $(\mathrm{n}=295), 28$-day mortality was $44.5 \%$ in the PMX-DHP group $(\mathrm{n}=147)$ and $43.9 \%$ in the sham group $(\mathrm{n}=148)(\mathrm{P}=0.092)$. In the PMX-DHP group, there was no significant difference in mean change of EAA between baseline and day 2 or 3 in all patients or among those on the MODS $>9$ groups. Dellinger et al. [32] commented in discussion that the relative timing, dose, and duration of PMX-DHP treatment may have been insufficient to reduce the endotoxin level and mortality.

In a post hoc analysis of EUPHRATES trial, Klein et al. [33] evaluated the impact of PMX-DHP on several endpoints in 194 septic shock patients with EAA level between 0.60 and 0.89: the PMX-DHP group $(\mathrm{n}=88)$ and the sham group $(\mathrm{n}=106)$. The 28-day mortality (26.1\%) in the PMX-DHP group was significantly $(\mathrm{P}=0.047)$ lower than that $(36.8 \%)$ in the sham group. Especially, patients with baseline norepinephrine $>0.1$ $\mu \mathrm{g} / \mathrm{kg} / \mathrm{min}$ had a significant $(\mathrm{P}=0.02)$ 28-day mortality reduction with PMX-DHP treatment $(17 / 69,24.6 \%)$ compared with sham $(34 / 85,40.0 \%)$. In addition, PMX-DHP significantly $(\mathrm{P}<0.05)$ increased median mean arterial pressure, median ventilator-free days to 28 days and survival to 90 days compared with sham. Accordingly, TIGRIS trial is currently underway in septic shock patients with MODS $>9$ and EAA level between 0.6 and 0.89 to elucidate the efficacy of PMX-DHP in these patients. 


\section{DURATION OF PMX-DHP}

Regarding the hemoperfusion period, the previous in vitro study using calf serum has demonstrated that the PMX-DHP could reduce the endotoxin level considerably in 2 hours of circulation [34], and this is the rationale for the 2 hours standard duration of PMX-DHP treatment. However, it remains unclear whether the optimal duration is 2 hours. We compared plasma levels of soluble adhesion molecules in 16 septic shock patients in the PMX-DHP greater than 2-hour (prolonged) group and the PMX-DHP 2-hour (conventional) group [14]. The norepinephrine dose, plasma levels of soluble endothelial leukocyte adhesion molecule- 1 and soluble intercellular adhesion molecule-1 significantly $(\mathrm{P}<0.05)$ decreased in the prolonged group than in the conventional group. Changes in $\mathrm{PaO}_{2} / \mathrm{FiO}_{2}$ ratio and SOFA score were significantly $(\mathrm{P}<0.05)$ improved in the prolonged group than in the conventional group. Yamashita et al. [35] have also shown that longer duration of PMX-DHP improved hemodynamics and pulmonary oxygenation in 37 patients with septic shock. Miyamoto et al. [36] have reported in their retrospective study that 12-hour duration of PMX-DHP $(\mathrm{n}=18)$ significantly increased mean arterial pressure and decreased the vasopressor dependency index compared with 2-hour duration of PMX-DHP $(n=18)$. Also, Kawazoe et al. [37] have demonstrated in a sub-analysis of the DESIRE (Dexmedetomidine for Sepsis in Intensive Care Unit Randomized Evaluation) trial, that the 28-day mortality rate was $7 / 22(31.8 \%)$ in the standard group and $0 / 14$ $(0 \%)$ in the extended group. Although these are all observational studies, longer duration of PMX-DHP have beneficial effects on hemodynamics in patients with septic shock. Furthermore, we have evaluated the extent of endotoxin adsorption by PMX-DHP performed for a 24-hour treatment period in 19 septic shock patients [38]. The plasma endotoxin levels of blood drawn from the radial artery and from the outlet circuit of the PMX-DHP column were measured by kinetic turbidimetric limulus assay using an MT-358 Toxinometer (Wako Pure Chemical Industries, Osaka, Japan) after 24 hours of PMX-DHP treatment. The endotoxin removal rate was defined by the following equation: [(radial artery endotoxin level-outlet circuit of PMX column endotoxin level)/radial artery endotoxin level $] \times 100 \%$. The median removal rate of endotoxin was $74.4 \%$. These findings suggested that 24-hour PMX-DHP treatment could remove endotoxin continuously throughout the entire treatment period.

\section{COST-EFFECTIVENESS OF PMX-DHP FOR SEPTIC SHOCK}

Berto et al. [39] conducted retrospective cost-effectiveness analysis of EUPHAS trial, which compared PMX-DHP versus conventional therapy based on calculated costs and survival years per patient per treatment arm. Direct medical costs included the consumption of hospital days, ICU days, catecholamine treatment days, renal replacement therapy days, mechanical ventilation treatment days, and the use of the PMXDHP device. All-cause hospital mortality was extrapolated to survival as expected life years per patient/arm. Life expectancy per patient in each group was calculated as the combination of life expectancy from Italian National Statistics Institute life tables and intrahospital mortality detected in the EUPHAS trial. Results of the base-case cost-effectiveness analysis were confirmed by all sensitivity analyses, with incremental costeffectiveness ratio values below commonly accepted value threshold. As a result, PMX-DHP could be considered a costeffective intervention for treatment of severe abdominal sepsis and septic shock. This finding suggests that PMX-DHP is a valuable treatment for septic shock.

\section{CONCLUSIONS}

Endotoxin adsorption therapy by PMX-DHP has been used for the treatment of septic shock patients. PMX-DHP directly adsorbed not only endotoxin but also monocytes and anandamide. PMX-DHP reduced blood levels of inflammatory cytokines such as IL-1, IL-6, TNF-alpha, and IL-17A, adhesion molecules, plasminogen activator inhibitor 1, and HMGB-1. Consequently, PMX-DHP increased blood pressure and reduced the dose of vasoactive-inotropic agents. Most of the prospective multicenter RCT of PMX-DHP could not show significant reduction in the 28-day mortality compared with the control group. One possible explanation for this failure is heterogeneity across patients fulfilling the diagnostic criteria for septic shock. However, a post hoc analysis of EUPHRATES trial has shown that PMXDHP significantly reduced the 28-day mortality in septic shock patients with EAA level between 0.60 and 0.89 . Longer duration of PMX-DHP may be another strategy to bring out the efficacy of PMX-DHP in patients with septic shock.

\section{CONFLICT OF INTEREST}

No potential conflict of interest relevant to this article was reported. 


\section{ACKNOWLEDGMENTS}

The authors would like to thank Go Murayama and Takuya Nemoto for technical assistance as a staff members of apheresis unit of Juntendo University Hospital.

\section{ORCID}

Chieko Mitaka

https://orcid.org/0000-0001-5671-1168

Makio Kusaoi https://orcid.org/0000-0003-0064-4765

Izumi Kawagoe https://orcid.org/0000-0002-5791-2700

Daizoh Satoh

\section{AUTHOR CONTRIBUTIONS}

Conceptualization: CM. Data curation: MK, IK. Methodology \& Project administration: CM, DS. Visualization \& Writingoriginal draft: CM. Writing-review \& editing: CM, DS.

\section{REFERENCES}

1. Aderem A, Ulevitch RJ. Toll-like receptors in the induction of the innate immune response. Nature 2000;406:782-7.

2. Reinhart K, Bayer O, Brunkhorst F, Meisner M. Markers of endothelial damage in organ dysfunction and sepsis. Crit Care Med 2002;30(5 Suppl):S302-12.

3. Tsujimoto H, Ono S, Hiraki S, Majima T, Kawarabayashi N, Sugasawa $\mathrm{H}$, et al. Hemoperfusion with polymyxin B-immobilized fibers reduced the number of CD16+ CD14+ monocytes in patients with septic shock. J Endotoxin Res 2004;10:229-37.

4. Nishibori M, Takahashi HK, Katayama H, Mori S, Saito S, Iwagaki $\mathrm{H}$, et al. Specific removal of monocytes from peripheral blood of septic patients by polymyxin B-immobilized filter column. Acta Med Okayama 2009;63:65-9.

5. Wang Y, Liu Y, Sarker KP, Nakashima M, Serizawa T, Kishida A, et al. Polymyxin B binds to anandamide and inhibits its cytotoxic effect. FEBS Lett 2000;470:151-5.

6. Nakamura T, Ebihara I, Shoji H, Ushiyama C, Suzuki S, Koide H. Treatment with polymyxin B-immobilized fiber reduces platelet activation in septic shock patients: decrease in plasma levels of soluble P-selectin, platelet factor 4 and beta-thromboglobulin. Inflamm Res 1999;48:171-5.

7. Nemoto H, Nakamoto H, Okada H, Sugahara S, Moriwaki K, Arai M, et al. Newly developed immobilized polymyxin B fibers improve the survival of patients with sepsis. Blood Purif 2001;19:361-8.

8. Nakamura T, Matsuda T, Suzuki Y, Shoji H, Koide H. Polymyx-

in B-immobilized fiber hemoperfusion in patients with sepsis. Dial Tranplant 2003;32:602-7.

9. Vincent JL, Laterre PF, Cohen J, Burchardi H, Bruining H, Lerma FA, et al. A pilot-controlled study of a polymyxin B-immobilized hemoperfusion cartridge in patients with severe sepsis secondary to intra-abdominal infection. Shock 2005;23: 400-5.

10. Cruz DN, Antonelli M, Fumagalli R, Foltran F, Brienza N, Donati A, et al. Early use of polymyxin B hemoperfusion in abdominal septic shock: the EUPHAS randomized controlled trial. JAMA 2009;301:2445-52.

11. Suzuki H, Nemoto H, Nakamoto H, Okada H, Sugahara S, Kanno $\mathrm{Y}$, et al. Continuous hemodiafiltration with polymyxin-B immobilized fiber is effective in patients with sepsis syndrome and acute renal failure. Ther Apher 2002;6:234-40.

12. Tani T, Hanasawa K, Kodama M, Imaizumi H, Yonekawa M, Saito M, et al. Correlation between plasma endotoxin, plasma cytokines, and plasminogen activator inhibitor- 1 activities in septic patients. World J Surg 2001;25:660-8.

13. Ikeda T, Ikeda K, Nagura M, Taniuchi H, Matsushita M, Kiuchi S, et al. Clinical evaluation of PMX-DHP for hypercytokinemia caused by septic multiple organ failure. Ther Apher Dial 2004;8:293-8.

14. Mitaka C, Tsuchida N, Kawada K, Nakajima Y, Imai T, Sasaki S. A longer duration of polymyxin B-immobilized fiber column hemoperfusion improves pulmonary oxygenation in patients with septic shock. Shock 2009;32:478-83.

15. Nakamura T, Sato E, Fujiwara N, Kawagoe Y, Maeda S, Yamagishi S. Suppression of high-mobility group box-1 and receptor for advanced glycation end-product axis by polymyxin B-immobilized fiber hemoperfusion in septic shock patients. J Crit Care 2011;26:546-9.

16. Sugiura M, Mitaka C, Haraguchi G, Tomita M, Inase N. Polymyxin B-immobilized fiber column hemoperfusion mainly helps to constrict peripheral blood vessels in treatment for septic shock. J Intensive Care 2015;3:14.

17. Nakamura T, Kawagoe Y, Matsuda T, Koide H. Effect of polymyxin B-immobilized fiber on bone resorption in patients with sepsis. Intensive Care Med 2004;30:1838-41.

18. Kushi H, Miki T, Okamaoto K, Nakahara J, Saito T, Tanjoh K. Early hemoperfusion with an immobilized polymyxin B fiber column eliminates humoral mediators and improves pulmonary oxygenation. Crit Care 2005;9:R653-61.

19. Ueno T, Ikeda T, Ikeda K, Taniuchi H, Suda S, Yeung MY, et al. HMGB-1 as a useful prognostic biomarker in sepsis-induced organ failure in patients undergoing PMX-DHP. J Surg Res 2011;171:183-90. 
20. Kushi H, Miki T, Nakahara J, Okamoto K, Saito T, Tanjoh K. Hemoperfusion with an immobilized polymyxin B column reduces the blood level of neutrophil elastase. Blood Purif 2006;24:212-7.

21. Coudroy R, Payen D, Launey Y, Lukaszewicz AC, Kaaki M, Veber B, et al. Modulation by polymyxin-B hemoperfusion of inflammatory response related to severe peritonitis. Shock 2017;47:93-9.

22. Bosmann A, Ward PA. Therapeutic potential of targeting IL17 and IL-23 in sepsis. Clin Transl Med 2012;1:4.

23. Flierl MA, Rittirsch D, Gao H, Hoesel LM, Nadeau BA, Day $\mathrm{DE}$, et al. Adverse functions of IL-17A in experimental sepsis. FASEB J 2008;22:2198-205.

24. Cantaluppi V, Assenzio B, Pasero D, Romanazzi GM, Pacitti A, Lanfranco G, et al. Polymyxin-B hemoperfusion inactivates circulating proapoptotic factors. Intensive Care Med 2008;34: 1638-45

25. Bellomo R, Kellum JA, Ronco C. Defining and classifying acute renal failure: from advocacy to consensus and validation of the RIFLE criteria. Intensive Care Med 2007;33:409-13.

26. Ono S, Tsujimoto H, Matsumoto A, Ikuta S, Kinoshita M, Mochizuki H. Modulation of human leukocyte antigen-DR on monocytes and CD16 on granulocytes in patients with septic shock using hemoperfusion with polymyxin B-immobilized fiber. Am J Surg 2004;188:150-6.

27. Monneret G, Lepape A, Voirin N, Bohé J, Venet F, Debard AL, et al. Persisting low monocyte human leukocyte antigen-DR expression predicts mortality in septic shock. Intensive Care Med 2006;32:1175-83.

28. Srisawat N, Tungsanga S, Lumlertgul N, Komaenthammasophon C, Peerapornratana S, Thamrongsat N, et al. The effect of polymyxin B hemoperfusion on modulation of human leukocyte antigen DR in severe sepsis patients. Crit Care 2018;22: 279.

29. Wittkowski H, Sturrock A, van Zoelen MA, Viemann D, van der Poll T, Hoidal JR, et al. Neutrophil-derived S100A12 in acute lung injury and respiratory distress syndrome. Crit Care Med 2007;35:1369-75.

30. Takahashi G, Hoshikawa K, Matsumoto N, Shozushima T, Onodera C, Kan S, et al. Changes in serum S100A12 and sRAGE associated with improvement of the $\mathrm{PaO} 2 / \mathrm{FiO} 2$ ratio follow- ing PMX-DHP therapy for postoperative septic shock. Eur Surg Res 2011;47:135-40.

31. Payen DM, Guilhot J, Launey Y, Lukaszewicz AC, Kaaki M, Veber B, et al. Early use of polymyxin B hemoperfusion in patients with septic shock due to peritonitis: a multicenter randomized control trial. Intensive Care Med 2015;41:975-84.

32. Dellinger RP, Bagshaw SM, Antonelli M, Foster DM, Klein DJ, Marshall JC, et al. Septic shock and elevated endotoxin level: the EUPHRATES randomized clinical trial. JAMA 2018;320: 1455-63.

33. Klein DJ, Foster D, Walker PM, Bagshaw SM, Mekonnen H, Antonelli M. Polymyxin B hemoperfusion in endotoxemic septic shock patients without extreme endotoxemia: a post hoc analysis of the EUPHRATES trial. Intensive Care Med 2018; 44:2205-12.

34. Shoji H. Extracorporeal endotoxin removal for the treatment of sepsis: endotoxin adsorption cartridge (Toraymyxin). Ther Apher Dial 2003;7:108-14.

35. Yamashita C, Hara Y, Kuriyama N, Nakamura T, Nishida O. Clinical effects of a longer duration of polymyxin B-immobilized fiber column direct hemoperfusion therapy for severe sepsis and septic shock. Ther Apher Dial 2015;19:316-23.

36. Miyamoto K, Kawazoe Y, Kato S. Prolonged direct hemoperfusion using a polymyxin B immobilized fiber cartridge provides sustained circulatory stabilization in patients with septic shock: a retrospective observational before-after study. J Intensive Care 2017;5:19.

37. Kawazoe Y, Sato T, Miyagawa N, Yokokawa Y, Kushimoto S, Miyamoto K, et al. Mortality effects of prolonged hemoperfusion therapy using a polymyxin B-immobilized fiber column for patients with septic shock: a sub-analysis of the DESIRE trial. Blood Purif 2018;46:309-14.

38. Mitaka C, Fujiwara N, Yamamoto M, Toyofuku T, Haraguchi G, Tomita M. Polymyxin B-immobilized fiber column hemoperfusion removes endotoxin throughout a 24-hour treatment period. J Crit Care 2014;29:728-32.

39. Berto P, Ronco C, Cruz D, Melotti RM, Antonelli M. Cost-effectiveness analysis of polymyxin-B immobilized fiber column and conventional medical therapy in the management of abdominal septic shock in Italy. Blood Purif 2011;32:33140. 